\title{
Ash from Mt St Helens
}

from Robert A. Kennedy

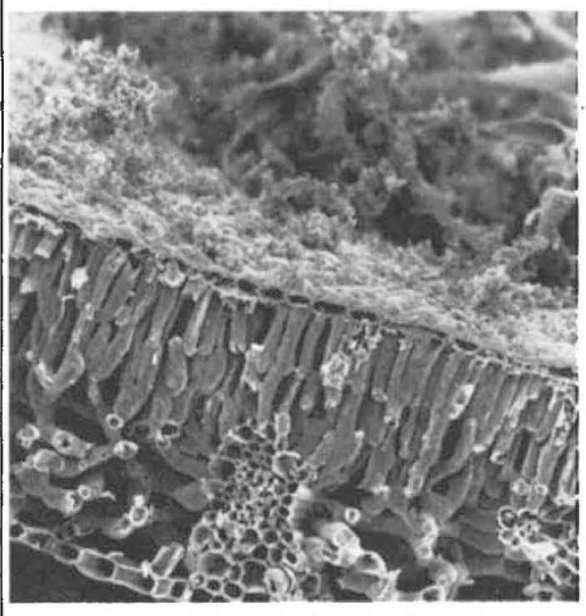

Cross section of apple leaf with ash on the upper epidermis.

AFTER the May 18th explosion of Mount Saint Helens ash fell on nearly seven million hectares of land in the State of Washington and in some places reached depths of $75 \mathrm{~cm}$. The pictures of plant leaves shown above were taken one and a half months after the explosion at Pullman, Washington, more than 250 miles from the volcano. Although over

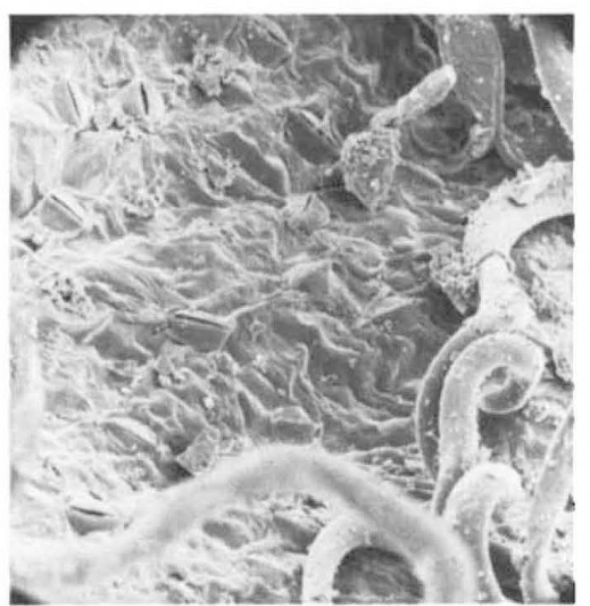

Lower apple leaf surface showing ash among the trichomes and stomata.

three inches of rain had fallen since the eruption large amounts of ash particles remain on the leaf surfaces, coating both the upper and lower epidermis and even finding their way into stomata.

The direct effect of the ash is to reduce light penetration; a one mm thick coating on the leaf absorbs 90 per cent of the light and virtually stops photosynthesis. More

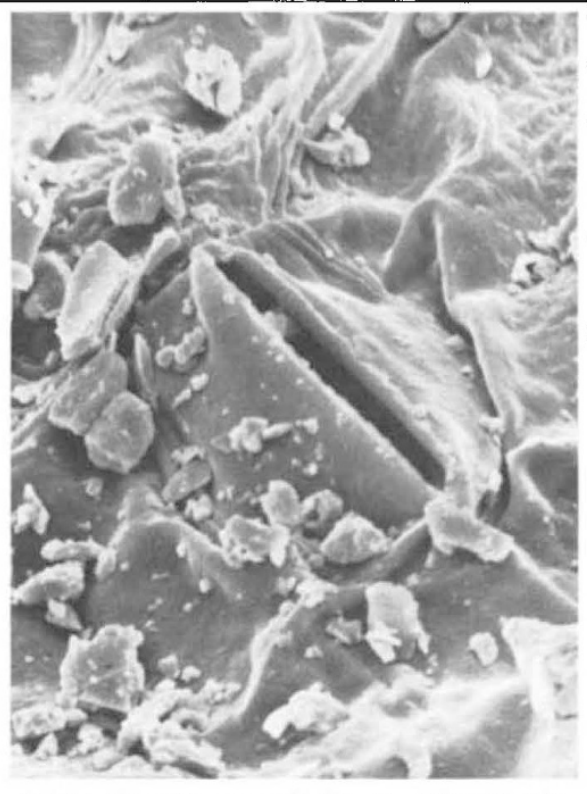

Ash inside a stoma on the lower epidermis of an apple leaf.

indirect and longer term effects may come from the presence in the ash (in concentrations of up to $10 \mathrm{ppm}$ ) of the toxic element fluoride.

Robert A. Kennedy is associate professor in the Department of Horticulture and Landscape Architecture, Washington State University. aperture defines the beam width, and hence the resolution. The second approach is the use of a Fresnel zone plate for focusing the radiation (G. Schmahl, University of Gottingen; N. M. Ceglio, Livermore Laboratories), a technique which has has resulted in $0.15 \mu \mathrm{m}$ resolution. The third method uses a focusing mirror with multi-layer coatings to increase reflectivity (E. Spiller, IBM New York; R. P. Haelbich, University of Hamburgh) and seems likely to yield the highest resolution.

The development of scanning acoustic microscopy (SAM) has two main objectives; the attainment of at least sub-optical resolution and the determination of the object's elastic constants. A sub-optical resolution microscope implies the use of a low-velocity, low-loss acoustic coupling liquid. Lemons (1974) has defined a coefficient of merit, $M$, which normalises the resolution attainable with a particular liquid to that with water - the liquid conventionally used in the SAM. Cryogenic liquids, for example, Argon at $87 \mathrm{~K}$ for which $M=3.7$ and Helium at $1.95 \mathrm{~K}$ and $0.4 \mathrm{~K}$ for which $M=3.7$ and 23 respectively offer potential improvements in SAM resolution. J. Heiserman (Stanford University) described a cryogenic reflection SAM designed to use these liquids and which currently works with liquid Helium down to $1.95 \mathrm{~K}$ where the wavelength is $360 \mathrm{~nm}$. The banded structure of human chromosomes has been clearly imaged with this system.
High pressure gases are, as pointed out by H. K. Wickramsinghe (University College, London), an attractive alternative to cryogenics since $M=4$ and 5 for Xenon and Argon at 40 and 250 atmospheres respectively. This suggests that resolutions just less than $100 \mathrm{~nm}$ can be achieved. Room temperature operation is an additional advantage that will allow simple sample preparation techniques and the imaging of living cells.

To measure an objects elastic constants C. F. Quate (Stanford University) described a technique in which the output voltage, $V$, of a reflection SAM is recorded as a function of defocusing distance, $z$, at a single image point. The $\mathrm{V}(z)$ curve obtained is a characteristic function of the elastic properties of the object surface. Both the density and the longitudinal velocity of the sample may, under certain conditions, be computed in this way.

The SAM has been used to ir age a wide range of objects. Coal may be characterised, as suggested by C. F. Quate, by its acoustic reflectivity. This technique should offer an increase in sensitivity of forty times over the comparable optical technique. $\mathrm{He}$ also showed using $\mathrm{V}(z)$ techniques that the SAM is eminently suitable for detecting delaminations in thin films such as chrome on glass. The large impedance mismatch between the surface of a metallic object and the water coupling the lens to the object may be overcome by replacing the water with a liquid metal such as mercury or gallium. This and the use of shear waves in addition to the liquid metal coupling can provide improved resolution due to the decreased wavelength and reduced spherical aberration in the object (J. Attal, Universitie des Sciences et Techniques du Languedoc).

Interest in the photoacoustic effect, first demonstrated by Bell in the 1880's, has increased dramatically in the last decade with the development of several photoacoustic imaging systems capable of microscopic resolution. In what has become conventional photoacoustic microscopy (PAM) a modulated light beam is scanned across a solid surface. A microphone is used to detect the pressure waves generated in the gas above the surface by virtue of the periodic heating at the sample surface. Since a lock-in amplifier is normally used in the detection system both the amplitude and phase of the photoacoustic signal can be mapped out across the sample. A. Rosencwaig (University of California) and G. Busse (ZWE Physik) pointed out the components of the signal detected at different phases relate to information from different depths within the sample. The system resolution is determined partly by the optical spot size and partly by the thermal wavelength in the solid. This latter quantity is inversely proportional to the square root of the beam modulation frequency and affects the depth of penetration of the thermal wave in 